\title{
Expression of an estrogen receptor variant lacking exon 3 in derivatives of MCF-7 cells with acquired estrogen independence or tamoxifen resistance
}

\author{
Feng Han, Richard Miksicek ${ }^{\mathbf{1}}$, Robert Clarke $^{\mathbf{2}}$ and Susan E Conrad \\ Department of Microbiology and Molecular Genetics, 2209 BPS, Michigan State University, East Lansing, Michigan 48824, USA \\ ${ }^{1}$ Department of Physiology, Michigan State University, East Lansing, Michigan 48824, USA \\ 'Lombardi Cancer Center and Department of Physiology and Biophysics, Georgetown University Medical School, Washington, DC 20007, USA \\ (Requests for offprints should be addressed to S Conrad; Email: conrad@msu.edu)
}

\begin{abstract}
The estrogen receptor (ER) plays important roles in the development and progression of breast cancer, and is a major target for tumor therapy. In this study, we investigated ER function in two derivatives of MCF-7 cells that were selected for their ability to proliferate in the absence of estrogen or in the presence of the antiestrogen, tamoxifen. Reporter gene assays indicated decreased ER activity in both cells lines, although the activity remaining retained responsiveness to both estrogen and tamoxifen. The decreased ER activity correlated with expression of a $61 \mathrm{kDa}$ variant ER protein, and sequencing of RT-PCR products indicated that this variant was the product of an exon 3 deletion (ER $\Delta E 3)$. To study its effects on cell proliferation, ER $\triangle \mathrm{E} 3$ cDNA was stably transfected into both the MCF-7 cell line and its estrogen-independent/tamoxifen-sensitive derivative MCF-7/LCC1 (LCC1), and the phenotypes of transfectants were examined. Expression of ER $\Delta \mathrm{E} 3$ was not sustainable in MCF-7 cells, but was maintained for at least 17 passages in LCC1 cells. These results are in agreement with previous reports that $\mathrm{ER} \triangle \mathrm{E} 3$ inhibits wild-type ER activity and negatively regulates proliferation of MCF-7 cells. They further suggest that the alteration that leads to estrogen independence in LCC1 cells allows for sustained expression of $\mathrm{ER} \triangle \mathrm{E} 3$, and that additional changes are required to confer tamoxifen resistance to these cells.
\end{abstract}

Journal of Molecular Endocrinology (2004) 32, 935-945

\section{Introduction}

Numerous studies have demonstrated the critical role that estrogen plays in the initiation and progression of breast tumors (Soule \& McGrath 1980, Henderson et al. 1988). Based on these findings, endocrine therapies have been developed and used for the treatment of breast cancer patients. Tamoxifen is the most commonly used endocrine therapy in hormone-responsive breast cancer, especially as adjuvant therapy after removal of the primary tumor (Jordan 2000). Worldwide clinical trials indicate that the 5- and 10-year mortality rates of breast cancer patients can be reduced 20-25\% by tamoxifen treatment (Early Breast Cancer Trialists' Collaborative Group 1992). Nevertheless, many tumors that initially respond to tamoxifen therapy eventually become resistant, and the development of such resistance in advanced breast cancer is a common cause for treatment failure. Recent reports indicate that aromatase inhibitors, which block production of estrogens, may be as or more effective than tamoxifen for the treatment of primary breast cancer (Milla-Santos et al. 2003). However, breast cancer cells can become estrogen independent, and the development of aromatase-resistant tumors might therefore occur. Understanding the molecular changes that take place during the evolution of resistance to endocrine therapies could provide strategies for preventing or treating such tumors. Several mechanisms might account for the development of resistance, including loss of estrogen receptor (ER), mutation of ER, alteration 
of ER cofactor(s), abnormal metabolism, and alterations in growth factor pathways (Osborne et al. 1995, Jordan 1998, Clarke et al. 2001a). ER mutations have been observed in some cell lines selected in vitro for resistance to antiestrogens, as well as in tumor samples (McGuire et al. 1992, Wolf \& Jordan 1994). However, many estrogenindependent and/or antiestrogen-resistant cell lines still express wild-type (wt) ER at significant levels (Jiang et al. 1992), suggesting that other mechanisms also exist.

Cell lines selected for the ability to grow in the absence of estrogen or in the presence of antiestrogen have been used as laboratory models of acquired endocrine resistance. MCF-7 cells have been widely used as a model of estrogen-dependent and antiestrogen-sensitive breast cancer. Both estrogen-independent and tamoxifen-resistant derivatives of MCF-7 have been selected using a protocol that mimics disease progression in patients (Leonessa et al. 1992, Brunner et al. 1993a,b, Thompson et al. 1993). MCF-7 cells were inoculated into ovariectomized athymic nude mice to select for estrogen-independent growth in vivo (Clarke et al. 1989). LGG1 cells were isolated from a rare tumor that developed, and were characterized as estrogen independent but tamoxifen sensitive (Brunner et al. 1993a). LCG1 cells were further selected in vitro for growth in the presence of 4-hydroxytamoxifen (4-OH TAM), giving rise to the MCF-7/LCG2 (LCG2) cell line, which is resistant to tamoxifen but still sensitive to steroidal antiestrogens such as ICI 182,780 (Brunner et al. 1993b). The phenotype of LCC2 cells is similar to that seen in many breast cancer patients who develop tamoxifen resistance during endocrine therapy (Howell et al. 1995).

The current study was aimed at identifying biochemical changes that occurred during the evolution of LCG1 and LCG2 cells. Estrogen response element (ERE) reporter gene assays demonstrated that although ER activity in LCG1 and LCG2 cells was regulated by 17- $\beta$ estradiol and 4-OH TAM, the overall level of expression of ERE reporter genes was significantly lower in LCG1 than in MCF-7 cells, and even lower in LCC2 cells. Western blot analyses revealed a $61 \mathrm{kDa}$ ER variant expressed in LCG1 and at higher levels in LCC2 cells, and sequencing of RT-PGR products identified this variant as one containing a deletion of exon 3. The $\mathrm{ER} \alpha$ gene has eight coding exons, which generate a $6 \cdot 2 \mathrm{~kb}$ wtER mRNA. Aberrant mRNA splicing can produce ER variant mRNAs with various exon deletions (Wang \& Miksicek 1991, Koehorst et al. 1993, Miksicek et al. 1993, Sluyser 1994, Gotteland et al. 1995, Pfeffer et al. 1995, Hopp \& Fuqua 1998, Murphy et al. 1998, Fasco et al. 2000). Variant ER mRNAs have been detected in normal breast tissue, breast cancer cell lines, and clinical tumor samples using RT-PCR. They are observed in the majority of ER-positive tumors and tumor cell lines, and attempts have been made to correlate changes in their expression with clinical features. In one study of breast tumors, expression of a specific ER variant correlated with antiestrogen resistance (Daffada et al. 1995). However, examination of in vitro-derived antiestrogen-resistant cell lines failed to observe any consistent changes in ER variant mRNA expression as a function of tamoxifen, ICI 164,384, or ICI 182,780 resistance (Madsen et al. 1997). In addition, although variant ER mRNAs are readily detected, the level of variant protein is usually extremely low or undetectable, making the physiological role of the RNAs questionable.

Among the characterized ER variants, one of the most commonly seen is the exon 3 deletion (ER $\Delta \mathrm{E} 3)$. Exon 3 encodes the second zinc finger of the DNA binding domain. The ER $\Delta \mathrm{E} 3$ protein does not bind to EREs and does not activate EREs in transient transfection assays, but can dimerize with both itself and wtER. When ER $\Delta \mathrm{E} 3$ was mixed with wtER in a ratio of $1: 1$, ERE activity was inhibited by $30 \%$, suggesting that ER $\Delta \mathrm{E} 3$ functions as a dominant negative variant (Wang \& Miksicek 1991). Consistent with this finding, ectopic expression of ERAE3 in MCF-7 cells has been reported to inhibit anchorage independent growth and to suppress their invasiveness (Erenburg et al. 1997). However, ER $\Delta \mathrm{E} 3$ has also been reported to be a potent activator of some promoters containing AP1 sites (Bollig \& Miksicek 2000), and it is therefore possible that it might promote proliferation under some circumstances.

The discovery that ER $\Delta \mathrm{E} 3$ protein is present in LCG1 and LCG2 cells suggested that they might be resistant to the inhibitory effects of this ER variant. Stable transfection experiments demonstrated that this was the case. Expression of ER $\Delta \mathrm{E} 3$ was strongly selected against in MCF-7 cells, but not in LCG1 cells. This is consistent with a model in which a change has occurred in LCG1 cells that 
makes them partially independent of ER function. However, this independence is not complete, since both LCG1 cells and derivatives expressing high levels of $\mathrm{ER} \Delta \mathrm{E} 3$ retain sensitivity to tamoxifen.

\section{Materials and methods}

\section{Cell culture}

MCF-7, LCG1 and LCG2 cells were maintained in improved modified Eagle's medium (IMEM) (Biofluids, Inc., Rockville, MD, USA) supplemented with 5\% fetal bovine serum (Hyclone Logan, Utah, USA), penicillin (100 units/ml) and streptomycin $(100 \mu \mathrm{g} / \mathrm{ml})$ (Invitrogen Life Technologies). To study the effects of estrogen and antiestrogens, cells were grown in IMEM without phenol red supplemented with 5\% charcoalstripped fetal bovine serum (CSS) (Hyclone).

\section{Reagents and plasmids}

$17 \beta$-Estradiol and 4-OH TAM were purchased from Sigma (Sigma Aldrich, St Louis, MO, USA). ICI 182,780 (ICI) was from AstraZeneca. Lipofectin was purchased from Life Technologies. The ER $\Delta E 3$ and wtER expression vectors (pCDNA3-ER $\Delta \mathrm{E} 3$ and pCDNA3-wtER $\alpha$ ) were constructed by insertion of the ER $\Delta \mathrm{E} 3$ and wtER $\alpha$ cDNAs into pCDNA3 (Invitrogen). pßgal-Basic was purchased from Clontech Laboratories (Palo Alto, CA, USA). The ERE-luciferase reporter construct, ERE2-tk109-luc, was obtained from Dr Gehm at Northwestern University Medical School (Gehm et al. 1997).

\section{Primers and PCR conditions}

The primers used for RT-PCR were 5'-CTGCGA AGGAGACTCGCTAC-3' (upstream) and 5'-AAG GCACTGACGATCTGGTG-3' (downstream). The primers used for genomic PCR were 5'-CGCTCG AGTGGGGTGCAACGTAGTAAGA-3' (upstream) and 5'-GCGAATTCGAATGGGTAGAGCGAG-3' (downstream). For reverse transcription reactions, $5 \mu \mathrm{g}$ total RNA and $10 \mathrm{pmol}$ of the downstream primer were incubated for $10 \mathrm{~min}$ at $65^{\circ} \mathrm{C}$ in deionized $\mathrm{H}_{2} \mathrm{O}$, and then in a total of $20 \mu \mathrm{l}$ reverse transcription mixture $(50 \mathrm{mM}$ Tris- $\mathrm{HCl}, \mathrm{pH} 8 \cdot 3$, $40 \mathrm{mM} \mathrm{KCl}, 6 \mathrm{mM} \mathrm{MgCl}$, $1 \mathrm{mM}$ dithiothreitol (DTT), $50 \mu \mathrm{M}$ dNTPs, 200 units MMLV-Reverse Transcriptase (Invitrogen Life Technologies) for
$1 \mathrm{~h}$ at $37^{\circ} \mathrm{C}$. For PCRs, $4 \mu \mathrm{l}$ of the reverse transcription mixture or $1 \mu \mathrm{g}$ genomic DNA were amplified in a final volume of $100 \mu \mathrm{l}$ containing $250 \mathrm{nM}$ of each primer, $200 \mu \mathrm{M}$ dNTPs, $1.5 \mathrm{mM}$ $\mathrm{MgCl}_{2}$, and $2 \cdot 5$ units TAQ DNA polymerase (Invitrogen Life Technologies). Each RT-PCR consisted of 35 cycles $\left(60 \mathrm{~s}\right.$ at $62{ }^{\circ} \mathrm{C}, 60 \mathrm{~s}$ at $74{ }^{\circ} \mathrm{C}$ and $30 \mathrm{~s}$ at $94^{\circ} \mathrm{C}$ ). Each genomic PCR consisted of 40 cycles $\left(60 \mathrm{~s}\right.$ at $58{ }^{\circ} \mathrm{C}, 60 \mathrm{~s}$ at $74^{\circ} \mathrm{C}$ and $60 \mathrm{~s}$ at $94^{\circ} \mathrm{C}$ ). PCR products were visualized on agarose gels stained with ethidium bromide. The DNA fragments of interest were then recovered and purified using a GenElute Agarose Spin Column (Sigma), and sequenced at the Michigan State University Genomic Technology Support Facility.

\section{Western blotting}

To prepare cell extracts, monolayers $(80 \%$ confluent) were washed twice in ice-cold PBS and collected by scraping and centrifugation. Cell pellets were resuspended in ice-cold lysis buffer (50 mM HEPES, $150 \mathrm{mM} \mathrm{NaCl,} \mathrm{pH} \mathrm{7.5,} 1 \mathrm{mM}$ EDTA, 2.5 mM EGTA, 0.1\% Tween-20, 10\% glycerol, $1 \mathrm{mM}$ DTT, $0 \cdot 1 \mathrm{mM}$ PMSF, $10 \mu \mathrm{g} / \mathrm{ml}$ leupeptin, $2 \mu \mathrm{g} / \mathrm{ml}$ aprotinin, $10 \mathrm{mM} \beta$-glycerophosphate and $1 \mathrm{mM} \mathrm{NaF}$ ) and lysed by sonication on ice (duty cycle $80 \%$, output 6, $10 \mathrm{~s}$ ) using a Sonifier 450 (Branson Ultrasonics Corporation, Danbury, CT, USA). The cellular debris was removed by centrifugation $\left(12000 \mathrm{~g}, 2 \mathrm{~min}, 4^{\circ} \mathrm{C}\right)$. The total protein concentration in the cell lysate was measured using a Bradford assay (Bio-Rad Laboratories, Inc. Hercules, CA, USA). Twenty micrograms total protein from each sample were separated on $12 \%$ SDS-polyacrylamide gels and transferred to PVDF membranes (NEN Life Science Products, Perkin Elmer, Boston, USA) in transfer buffer $(25 \mathrm{mM}$ Tris, $192 \mathrm{mM}$ glycine and $15 \%$ methanol) using the XCELL gel transfer system (Invitrogen Life Technologies). Western analysis of ER protein levels utilized a mouse monoclonal antibody (Mab-17) that was raised against recombinant ER $\alpha$ protein, and which recognizes an amino-terminal epitope present in both wtER and ER $\Delta$ E3. Membranes were blocked in 5\% dry milk in PBST (PBS $+0 \cdot 1 \%$ Tween 20) and incubated with a 1:2 dilution of the Mab-17 in PBS at $4{ }^{\circ} \mathrm{C}$ overnight. The antibody was removed and the membrane was washed with PBST three times for $10 \mathrm{~min}$ each. A peroxidase-labeled 
secondary antibody (American Qualex, San Clemente, CA, USA) was diluted 1:2000 in 5\% dry milk in PBST and incubated with the membrane for $1 \mathrm{~h}$ at room temperature. Blots were reprobed with an antibody to $\beta$-actin as a loading control. Proteins were then visualized using Supersignal West Pico Chemiluminescent Substrate (Pierce, Rockford, IL, USA).

\section{Transient transfection and luciferase assays}

Cells were plated in IMEM containing 5\% FBS at $4 \times 10^{5}$ cells per $60 \mathrm{~mm}$ tissue culture plate, and incubated overnight. Each plate was then incubated with $5 \mu \mathrm{g}$ ERE2-tk109-luc and $1 \mu \mathrm{g}$ p $\beta$ galBasic using Lipofectin (Invitrogen Life Technologies) as the transfection agent. Lipofectin/ DNA complexes were formed in phenol red-free, serum-free IMEM, and left on plates for $6 \mathrm{~h}$. The transfection medium was then removed, cells were washed twice in PBS and then incubated in IMEM supplemented with 5\% CSS for $48 \mathrm{~h}$ to deprive them of estrogen. Fresh medium containing 5\% CSS with or without estrogen $\left(10^{-9} \mathrm{M}\right), 4-\mathrm{OH}$ TAM $\left(10^{-6} \mathrm{M}\right)$, or ICI $\left(10^{-7} \mathrm{M}\right)$ was then added for $8 \mathrm{~h}$. Treatments were added as stock solutions in absolute ethanol, and ethanol was added to control media to the same final concentration in all plates. After the $8 \mathrm{~h}$ treatment, cells were washed twice with PBS and harvested by scraping and centrifugation. Cell pellets were resuspended in $200 \mu \mathrm{l}$ reporter lysis buffer (Promega Corp., Madison, WI, USA) and lysed by freezing and thawing. Protein concentrations in cell extracts were determined using the Modified DC assay (Bio-Rad). Aliquots of each lysate were assayed for $\beta$-galactosidase (Clontech) and luciferase (Promega) activities on a Turner TD-20e luminometer using protocols suggested by the manufacturer. The luciferase activity was normalized to the $\beta$-galactosidase activity in the same extract, and is shown as the mean \pm S.D. of three independent transfections.

\section{Stable transfections}

To obtain stable cell lines, MCF-7 and LCG1 cells were transfected with pCDNA3-ER $\Delta 3$ or pCDNA3-wtER $\alpha$ using the Lipofectin reagent, and selected in medium containing $400 \mu \mathrm{g} / \mathrm{ml}$ G418 (Geneticin, Amersham BioSciences, Piscatawy, NJ, USA). Individual G418 resistant colonies were isolated, and expanded into cell lines that were subsequently maintained in media containing $40 \mu \mathrm{g} / \mathrm{ml} \mathrm{G418.} \mathrm{Passage} \mathrm{one} \mathrm{was} \mathrm{defined} \mathrm{as} \mathrm{the}$ point at which each transfected cell line was passed to a single $10 \mathrm{~cm}$ plate. Clonal cell lines that expressed ER $\Delta \mathrm{E} 3$ or wtER were identified by Western blotting.

\section{Colony formation in soft agar}

Cells were plated in phenol red-free IMEM containing 5\% C.SS for two days, then harvested by trypsinization. Cells were suspended in IMEM containing 5\% CSS, $0 \cdot 3 \%$ Nobel agar (Difco Laboratories, BDDiagnostic Systems, Sparks, MD, USA) in the presence or absence of estrogen or 4-OH TAM. They were then plated at a density of $5 \times 10^{4}$ cells/well in 6-well dishes on top of a layer of IMEM containing $5 \%$ C.SS plus or minus estrogen or $4-\mathrm{OH}$ TAM and $0 \cdot 6 \%$ agar. The plates were incubated for 21 days, with feeding every 5 days. Colonies were then stained with $0 \cdot 1 \%$ neutral red and analyzed by microscopy. Colonies larger than $60 \mu \mathrm{m}$ (more than 50 cells) were counted, and six independent wells per treatment were averaged. The results were expressed as means \pm S.D.

\section{Results}

\section{ER activity in MCF-7, LCC1 and LCC2 cells}

To investigate whether ER activity or regulation was altered in LCG1 or LCC2 cells, expression and regulation of an ERE-Luc reporter gene in these cell lines were compared with MCF-7 cells. An ERE-luciferase plasmid construct (ERE2-tk109-luc) was co-transfected into cells along with a control $\beta$-galactosidase construct (pßgal-Basic) as described in Materials and methods. After transfection, cells were treated with vehicle, estrogen, 4-OH TAM, or ICI for $8 \mathrm{~h}$, harvested and analyzed for luciferase and $\beta$-galactosidase activities as described in Materials and methods. As shown in Fig. 1, the adjusted luciferase activity was low in all three cell lines in CSS, although it was significantly higher in MCF-7 than in LCG1 $(P=0.0003)$ or LCG2 $(P<0.0001)$ cells. Estrogen induced luciferase expression, and this induction was reversed by both ICI and 4-OH TAM in all of the cell lines. This indicates that both estrogen and antiestrogens regulate ER activity in LCG1 and LCG2 cells. However, the magnitude of luciferase induction by 


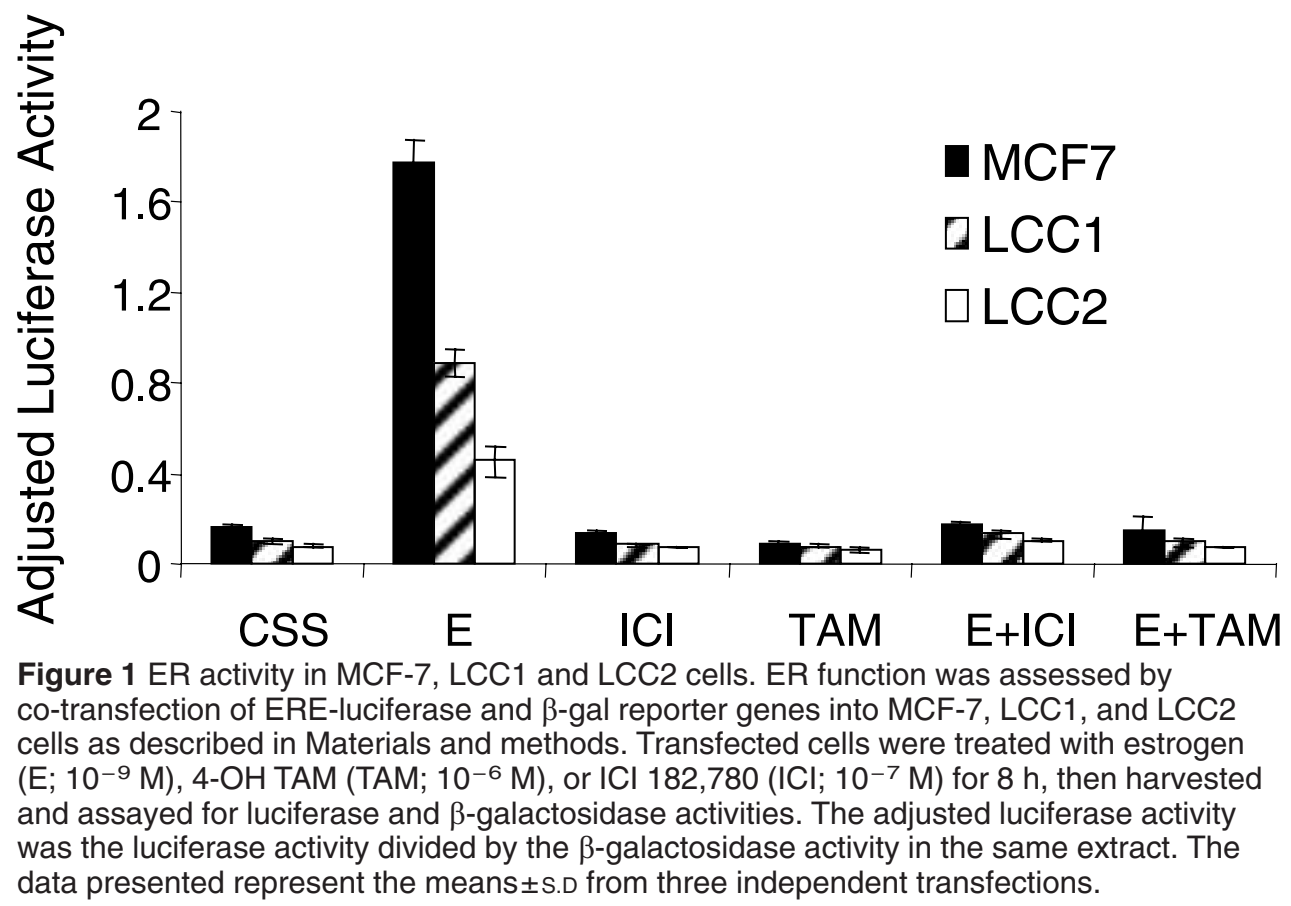

estrogen was significantly lower in LCC2 than in either MGF-7 $(P=0 \cdot 008)$ or LGG1 $(P=0 \cdot 0055)$ cells. Together with the lower basal ER activity in LCG1 and LCG2 cells, these results suggested that ER function might be altered in LCG1 and/or LCC2 cells, despite the fact that ER levels and binding affinities in these cell lines were previously shown to be comparable to MCF-7 cells by ligand binding assays (Brunner et al. 1993b).

\section{Identification of ER $\Delta$ E3 protein in LCC1 and LCC2 cells}

To investigate the cause of the altered ER activity in LCG1 and LCG2 cells, Western blot analyses of
ER $\alpha$ protein were carried out. MGF-7, LCG1 and LCC2 cells were incubated in IMEM/CSS for 2 days, and then treated with estrogen, 4-OH TAM, or ICI. After 2 days of treatment, cells were harvested and lysates were analyzed by Western blotting using a monoclonal antibody that recognizes an N-terminal epitope of wtER. ER expression levels were similar in the three cell lines (Fig. 2). Interestingly, an ER variant of approximately $61 \mathrm{kDa}$ was detected at high levels in LCC2, and to a lesser extent in LCG1, cells. In this experiment, the level of the $61 \mathrm{kDa}$ ER variant was comparable to that of the $66 \mathrm{kDa}$ wtER in LCC2 cells. In addition, expression of the $61 \mathrm{kDa} \mathrm{ER}$ variant was regulated by

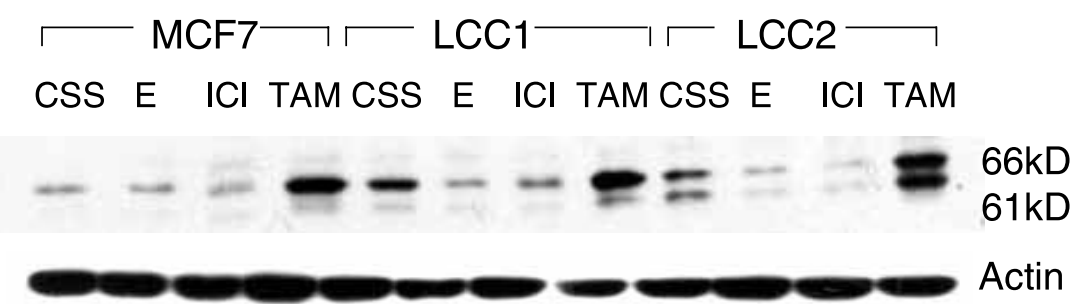

Figure 2 Analysis of ER protein expression in MCF-7, LCC1 and LCC2 cells. MCF-7, LCC1, and LCC2 cells were treated with estrogen (E), 4-OH TAM (TAM), or ICI 182,780 (ICl) for $48 \mathrm{~h}$. Cell extracts were prepared and analyzed for ER expression by SDS-polyacrylamide gel electrophoresis and Western blotting as described in Materials and methods. 
estrogen, 4-OH TAM and ICI in the same way as that of wtER.

To investigate the nature of the ER variant in LCC2 cells, it was molecularly characterized. Based on the size of the protein, it was suspected to arise from a deletion of exon 3 . To test this hypothesis, a set of primers was designed to span exon 3 , with the upstream primer located in exon 2 and the downstream in exon 4. Total RNA was isolated from MCF-7 and LGC2 cells, and RT-PGRs were performed as described in Materials and methods. Control PCRs were carried out in parallel with cDNA constructs containing either the wild-type or $\Delta 3 \mathrm{ER}$ coding sequences. As shown in Fig. 3a, RT-PCRs using MCF-7 cell RNA gave rise to a single band of the predicted $420 \mathrm{bp}$ that co-migrated with the fragment amplified from the wtER cDNA. This fragment was also present in the RT-PCR products from LCG2 cells; however, the LCG2 cell products also included a $320 \mathrm{bp}$ fragment that co-migrated with the fragment amplified from the $\mathrm{ER} \Delta \mathrm{E} 3 \mathrm{cDNA}$. To confirm that the $320 \mathrm{bp}$ fragment amplified from LCC2 cells indeed represented an exon 3 deletion, it was purified and sequenced. The sequencing results established that LCC2 cells contain significant amounts of an mRNA species containing a precise deletion of exon 3 (Fig. 3b), which is likely to account for the $61 \mathrm{kDa}$ ER variant protein detected by Western blotting.

One potential mechanism for the generation of the ERAE3 mRNA found in LCG2 cells would be a splice site mutation in one allele of the genomic DNA. To test for such a mutation, genomic PCR was carried out in MCF-7 and LCG2 cells using a primer set with the first strand primer located in intron 2 and the second in intron 3. The resulting 313 bp fragment containing exon 3 plus flanking intron sequences was then purified and sequenced. No genomic mutations were observed within 122 bp upstream or 77 bp downstream of exon 3 in either MCF-7 or LCC2 cells (data not shown), a region that typically includes all essential splicing signals including the branch point adenosine.

\section{Ectopic expression of ER $\Delta E 3$ in stably transfected MCF-7 and LCC1 cells}

Previous research has indicated that ERAE3 inhibits wtER function at EREs, and also inhibits both anchorage-independent growth and invasiveness of transfected MCF-7 cells. In addition,
A.
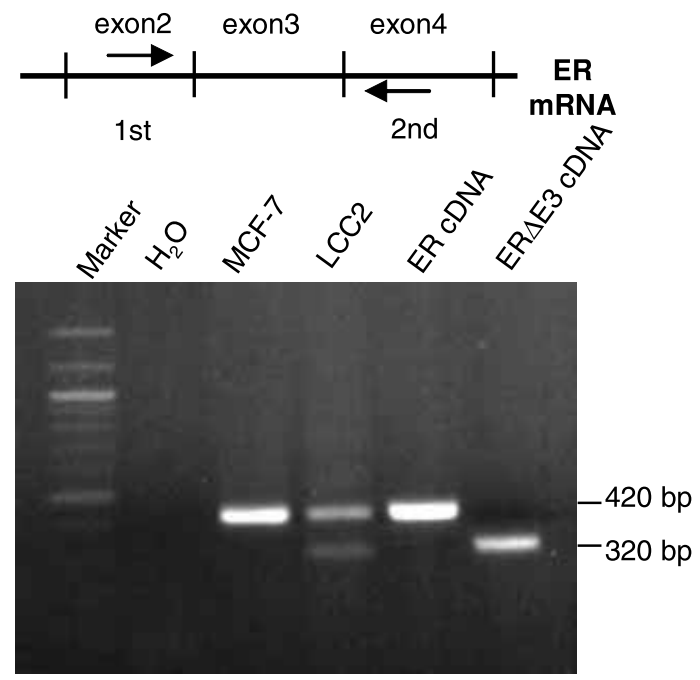

B.

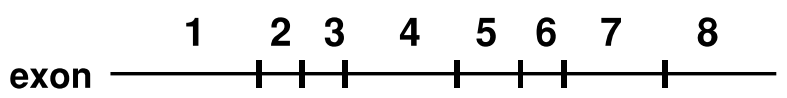

ER

\begin{tabular}{|l|l|l|l|}
\hline AF1 & DBD & LBD AF2 & \\
\hline
\end{tabular}

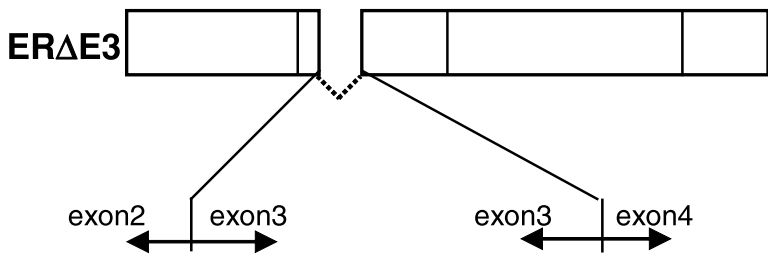

..TTCAAG GACATAA .... ATGAAAGGTGG GATAC...

Figure 3 Characterization of ER mRNAs expressed in LCC2 cells. (A) Total RNA was isolated from MCF-7 and LCC2 cells as described in Materials and methods. RT-PCRs were carried out using the indicated primers. PCRs with both wild-type ER and ER $\triangle E 3$ cDNAs were included as controls. Final PCR products were separated on agarose gels and stained with ethidium bromide. (B) The $420 \mathrm{bp}$ and $320 \mathrm{bp}$ PCR products from MCF-7 and LCC2 cells were purified and sequenced, and the results are illustrated diagrammatically. The sequences found in both the 420 and 320 bp products are shown in bold letters, and those found only in the $420 \mathrm{bp}$ fragment are shown in italics. DBD, DNA binding domain; LBD, ligand binding domain.

ERAE3 mRNA levels were higher in normal mammary epithelial cells than in breast cancer cells, suggesting that it may be involved in limiting 

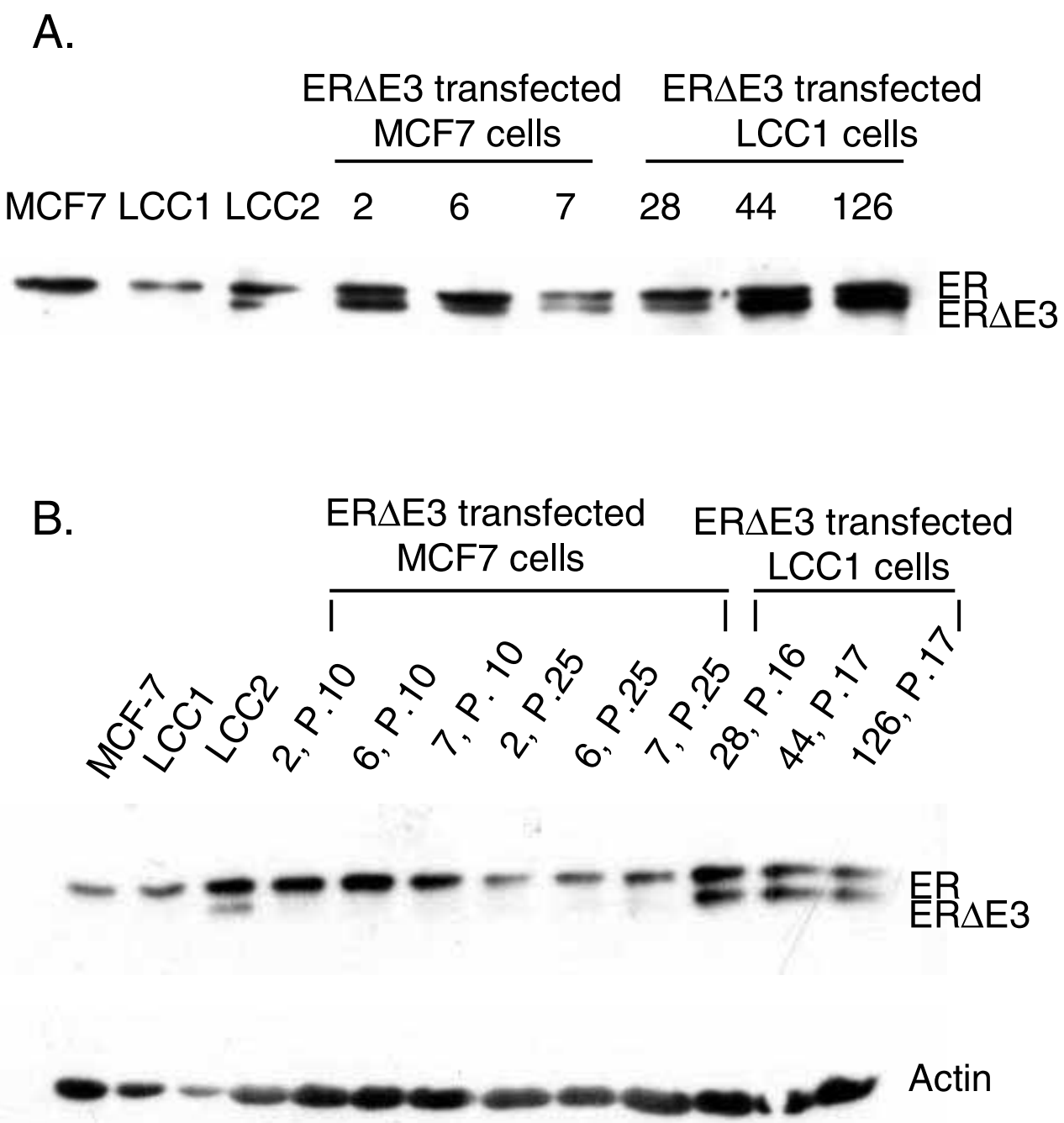

Figure 4 ER $\Delta$ E3 expression in stably transfected MCF-7 and LCC1 cells. MCF-7 and LCC 1 cells were transfected with wtER and ER $\triangle E 3$ CDNAs, and stable transfectants were selected as described in Materials and methods. (A) Western blot analysis of ER proteins expressed at the time of screening individual transfectants from each cell line. Lines 2, 6, and 7 represent three independent pCDNA3-ER $\triangle \mathrm{E} 3$ transfected MCF-7 cell clones. Lines 28, 44, and 126 represent three independent pCDNA3-ERAE3 transfected LCC1 cell clones. Western blots of extracts from MCF-7, LCC1 and LCC2 cells are included as controls. (B) Western blot analysis of the ER protein present in later passages (P.10, P.25, P.16, P.17) of the cell lines shown in (A).

proliferation (Erenburg et al. 1997). Given these facts, the high levels of ER $\Delta \mathrm{E} 3$ protein in LCC2 cells, which have increased tumorigenic potential relative to MCF-7 cells, was surprising. To investigate whether expression of $\mathrm{ER} \Delta \mathrm{E} 3$ has different consequences in MCF-7 cells and their estrogen-independent derivative, LCG1, stable transfections were carried out. MCF-7 and LCG1 cells were transfected with either pCDNA3-wtER or pCDNA3-ER $\Delta \mathrm{E} 3$, and G418 ${ }^{\mathrm{r}}$ colonies were selected and expanded into clonal cell lines. Lines expressing ER $\Delta \mathrm{E} 3$ or wtER were identified by RT-PGR and confirmed by Western blotting. As shown in Fig. 4a, when cultures were analyzed soon (within 1-2 passages) after selection, three independent derivatives of both MCF-7 and LGC1 
expressed the ER $\Delta \mathrm{E} 3$ gene at similar levels to the endogenous wild-type. However, later passages of the MCF-7-derived lines no longer expressed the $61 \mathrm{kDa}$ ER variant (Fig. 4b), suggesting that it had been selected against. In contrast, the LCG1derived lines maintained expression of the variant protein for at least 17 passages. These results indicate that a change occurred during the derivation of LCG1 cells that allows them to tolerate continued expression of ER $\Delta \mathrm{E} 3$.

\section{Anchorage-independent proliferation of LCC1 cells expressing ER $\Delta E 3$}

LCC2 cells consistently express higher levels of $\mathrm{ER} \Delta \mathrm{E} 3$ protein than LCG1 cells and have also acquired tamoxifen resistance. To investigate whether expression of ectopic ER $\Delta \mathrm{E} 3$ affected the growth properties and/or tamoxifen sensitivity of LCG1 cells, the ability of control and transfected cell lines to form colonies in soft agar were compared. Cell lines examined included MCF-7, LCG1, LCG2, LCG1 cells stably expressing ER $\triangle \mathrm{E} 3$ or wtER, and LCG1 cells containing the pcDNA3 vector. Expression of ER $\Delta \mathrm{E} 3$ and wtER in the transfected cell lines at the start of these assays was confirmed by Western blotting of cell lysates (Fig. 5a). The ability of the different cell lines to form colonies in soft agar after a 3-week incubation in medium containing CSS, CSS+ estrogen, or CSS+4-OH TAM was evaluated by staining with neutral-red followed by microscopy (Fig. 5b) and direct counting of colonies (Fig. 5c).

Figure 5 Effects of ER $\Delta E 3$ expression on the growth properties of LCC1 cells. (A) Expression of wtER and ER $\triangle E 3$ in transfected cell lines was confirmed by Western blotting. Lines 28, 44, and 126 represent three independent pCDNA3-ER $\triangle E 3$ transfected LCC1 cell clones. Lines 26 and 271 represent two independent pCDNA3-wtER $\alpha$ transfected LCC1 cell clones. Line 15 is a pCDNA3 transfected LCC1 cell clone. (B) Cells were plated in IMEM/CSS for 2 days and then grown in $0.3 \%$ soft agar with or without estrogen $\left(E ; 10^{-9} \mathrm{M}\right)$ or 4-OH TAM (TAM; $10^{-6} \mathrm{M}$ ) for 21 days as described in Materials and methods. LCC $1 / \mathrm{ER} \Delta \mathrm{E} 3$ represents data from clone 28, which was similar to clone 44 and clone 126. LCC1/wtER represents data from clone 26, which was similar to clone 271. LCC1/pCDNA3 represents clone 15's data. (C) Colonies larger than $60 \mu \mathrm{m}$ (more than 50 cells) were counted, and six independent wells per treatment per sample were averaged. The results are expressed as means \pm S.D
As shown in Fig. 5, colony formation by MCF-7 cells was significantly higher in the presence of estrogen than in the absence of estrogen or in the

A.
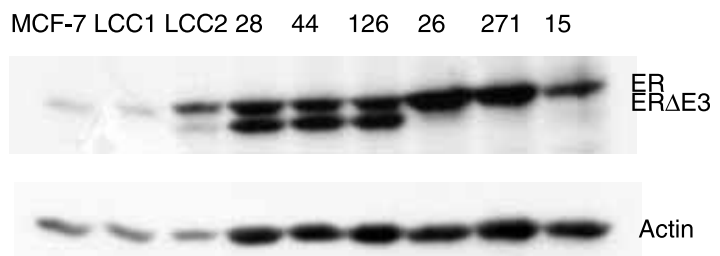

B.

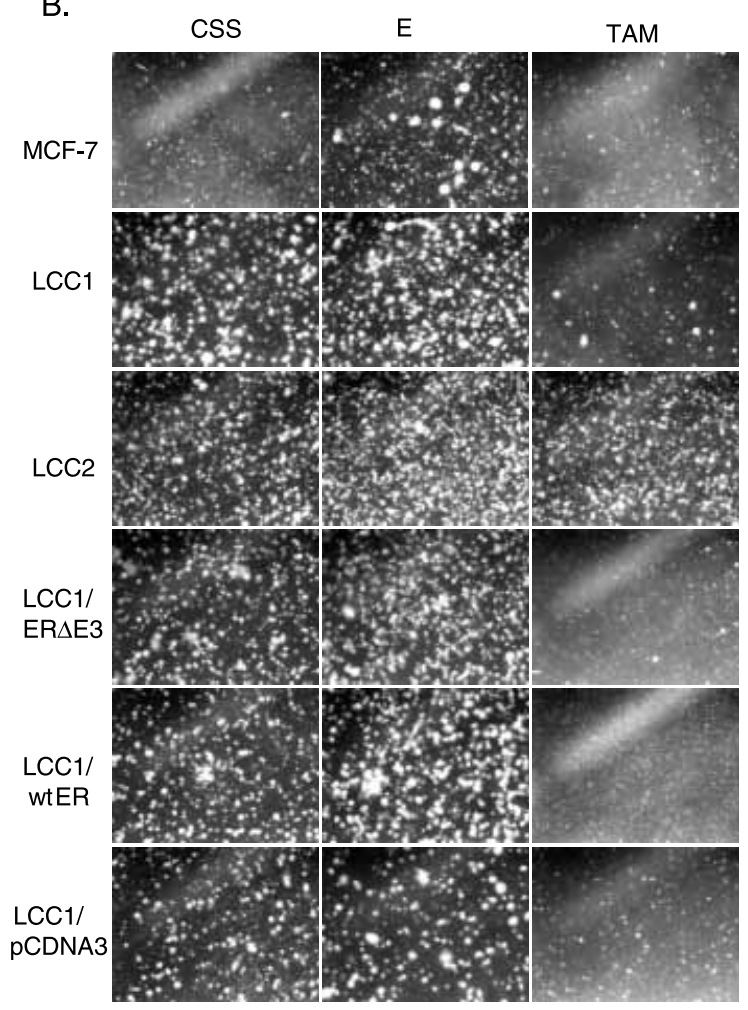

C.

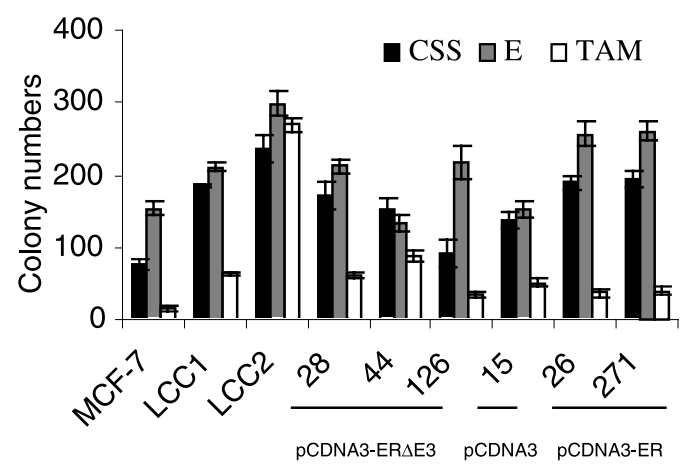

www.endocrinology.org 
presence of $4-\mathrm{OH}$ TAM $(P<0 \cdot 0001)$. Colony formation by LCG1 cells was similar in the presence or absence of estrogen, but low in the presence of $4-\mathrm{OH}$ TAM $(P<0 \cdot 0001)$. In contrast, LCG2 cells formed colonies with similar efficiency under all conditions. Thus, the behavior of these cell lines in the soft agar assay clearly reflects their reported phenotypes (Clarke et al. 2001a). The phenotype of LCG1 cells stably expressing either wtER $\alpha$ or ER $\Delta \mathrm{E} 3$ was unchanged; in each case the number of colonies formed in the presence of 4-OH TAM was significantly less than in CSS or CSS plus estrogen ( $P$ values ranged from 0.01 to $<0.0001$ ) (Fig. 5c). Thus, expression of ER $\Delta \mathrm{E} 3$ does not inhibit LCG1 colony formation in the absence or presence of estrogen, nor does it confer tamoxifen resistance to these cells. Because ER $\Delta \mathrm{E} 3$ expression was not maintained in transfected MCF-7 cells (Fig. 4b), we were unable to directly examine the effects of ER $\Delta \mathrm{E} 3$ expression on colony formation in this cell line. However, a previous report indicated that expression of ER $\Delta \mathrm{E} 3$ inhibits the ability of MCF-7 cells to proliferate in soft agar in the presence of estrogen (Erenburg et al. 1997), and this is consistent with the data presented here.

\section{Discussion}

In this study, we investigated ER expression and function in MCF-7-derived cell lines with acquired estrogen independence or tamoxifen resistance. We have shown that a specific ER variant, $\mathrm{ER} \Delta \mathrm{E} 3$, is expressed at high levels in the estrogenindependent/tamoxifen-resistant LCG2 cell line, and to a lesser extent in estrogen-independent but tamoxifen-sensitive LCG1 cells. Although there have been numerous reports of ER variant mRNAs in breast cancer cells (Wang \& Miksicek 1991, Koehorst et al. 1993, Miksicek et al. 1993, Sluyser 1994, Gotteland et al. 1995, Pfeffer et al. 1995, Hopp \& Fuqua 1998, Murphy et al. 1998, Fasco et al. 2000), the existence of variant proteins has been more controversial, and this is one of the few cases where a significant amount of a naturally occurring ER variant is expressed at the protein level.

The identification of an ER variant that is expressed at high levels in breast cancer cell lines raises several interesting questions. One is the mechanism leading to expression of the variant protein, and the second is its effects on cellular phenotype. Previous studies have shown that several different variant ER mRNAs can co-exist in both normal and transformed tissues (Gotteland et al. 1995), suggesting that they generally do not result from simple mRNA splice site mutations. By sequencing PCR products from genomic DNA, we demonstrated that the presence of ER $\Delta \mathrm{E} 3 \mathrm{mRNA}$ in LCC2 cells is not a result of a simple splice site mutation (data not shown). The analysis carried out does not, however, rule out the complete loss of exon 3 and surrounding sequences from one allele of the ER gene in LCC2 cells. Early evidence suggested that exon skipping is one of the likely sources of ER variant mRNAs in breast cancer cells (Miksicek et al. 1993). The amount of ER $\Delta \mathrm{E} 3$ protein present in LCG1 and LCC2 cells is somewhat variable (compare Figs 2 and 5), which is consistent with this suggestion. However, the amount of ER $\Delta 3$ protein is consistently higher in LCG2 than LCG1 cells, indicating that expression of this variant is regulated.

In addition to investigating the source of the increased ER $\Delta \mathrm{E} 3$ in LCC2 cells, we have characterized its effects upon cellular phenotype, and found that the increased level of ER $\Delta \mathrm{E} 3$ from MCF-7 to LCG1 to LCG2 cells correlates with decreased activity of ERE reporter genes in these cell lines. Furthermore, since its expression is not maintained in stably transfected MCF-7 cells, ER $\Delta \mathrm{E} 3$ seems to inhibit the estrogen-dependent proliferation or survival of these cells. These findings are consistent with previous reports that ER $\Delta \mathrm{E} 3$ acts as a dominant negative mutant at EREs (Wang \& Miksicek 1991, Erenburg et al. 1997, Bollig \& Miksicek 2000). In contrast to the situation in MCF-7 cells, ER $\Delta \mathrm{E} 3$ expression can be maintained for many passages in LGG1 cells. The fact that ER $\Delta \mathrm{E} 3$ does not inhibit proliferation of LCG1 cells suggests that these cells no longer require the action of ER at ERE-regulated genes, and it seems likely that the same change(s) giving rise to this phenotype also confer estrogen independence. Such changes might include activation of genes that are normally induced by estrogen, increased expression or mutation of ER coregulators, or activation of growth factor pathways (Clarke et al. 2001a,b).

It is interesting to note that although LCG1 cells proliferate in the presence of what appears to be a dominant negative ER, they are still sensitive to tamoxifen, indicating that they are not completely 
independent of ER function. There are several possible explanations for this result. One is that tamoxifen-bound ER may have unique effects upon some genes that regulate cell proliferation; for example it might suppress genes required for proliferation or activate genes that inhibit proliferation. Alternatively, ER $\Delta \mathrm{E} 3$ may activate genes that promote proliferation, either via interactions with transcription factors such as AP1, or via non-genomic mechanisms, and these activities may be inhibited by tamoxifen. Although our results indicate that such activities are not sufficient to promote proliferation, their inhibition might prevent it. Determining which, if either, of these explanations is correct would have important implications for understanding the mechanisms leading to tamoxifen resistance. Finally, although our data clearly demonstrate that expression of ER $\Delta E 3$ is not sufficient to confer tamoxifen resistance to LCG1 cells, it is possible that increased ER $\Delta \mathrm{E} 3$ expression plays an indirect or contributory role in its development. The continuous presence of $\mathrm{ER} \Delta \mathrm{E} 3$ may impose a selective pressure on LGG1 cells to become less dependent on ER function and, over time, may favor the emergence of tamoxifen-resistant progeny.

\section{Acknowledgements}

This work was partially supported by grants NIH CA76647 (S E G) and DAMD1794J4372 (R M).

\section{References}

Bollig A \& Miksicek RJ 2000 An estrogen receptor-alpha splicing variant mediates both positive and negative effects on gene transcription. Molecular Endocrinology 14 634-649.

Brunner N, Boulay V, Fojo A, Freter CE, Lippman ME \& Clarke R 1993a Acquisition of hormone-independent growth in MCF-7 cells is accompanied by increased expression of estrogen-regulated genes but without detectable DNA amplifications. Cancer Research 53 283-290.

Brunner N, Frandsen TL, Holst-Hansen C, Bei M, Thompson EW, Wakeling AE, Lippman ME \& Clarke R $1993 b$ MCF7/LCC2: a 4-hydroxytamoxifen resistant human breast cancer variant that retains sensitivity to the steroidal antiestrogen ICI 182 780. Cancer Research 53 3229-3232.

Clarke R, Brunner N, Katzenellenbogen BS, Thompson EW, Norman MJ, Koppi C, Paik S, Lippman ME \& Dickson RB 1989 Progression of human breast cancer cells from hormone-dependent to hormone-independent growth both in vitro and in vivo. PNAS 86 3649-3653.
Clarke R, Leonessa F, Welch JN \& Skaar TC 2001 $a$ Cellular and molecular pharmacology of antiestrogen action and resistance. Pharmacological Reviews 53 25-71.

Clarke R, Skaar TC, Bouker KB, Davis N, Lee YR, Welch JN \& Leonessa F 2001 $b$ Molecular and pharmacological aspects of antiestrogen resistance. Fournal of Steroid Biochemistry and Molecular Biology 76 71-84.

Daffada AA, Johnston SR, Smith IE, Detre S, King N \& Dowsett M 1995 Exon 5 deletion variant estrogen receptor messenger RNA expression in relation to tamoxifen resistance and progesterone receptor/pS2 status in human breast cancer. Cancer Research $\mathbf{5 5}$ 288-293.

Early Breast Cancer Trialists' Collaborative Group 1992 Systemic treatment of early breast cancer by hormonal, cytotoxic, or immune therapy. 133 randomised trials involving 31000 recurrences and 24000 deaths among 75000 women. Lancet 339 $71-85$.

Erenburg I, Schachter B, Mira y Lopez R \& Ossowski L 1997 Loss of an estrogen receptor isoform (ER alpha delta 3) in breast cancer and the consequences of its reexpression: interference with estrogen-stimulated properties of malignant transformation. Molecular Endocrinology $112004-2015$.

Fasco MJ, Keyomarsi K, Arcaro KF \& Gierthy JF 2000 Expression of an estrogen receptor alpha variant protein in cell lines and tumors [In Process Citation]. Molecular and Cellular Endocrinology $162167-180$.

Gehm BD, McAndrews JM, Chien PY \& Jameson JL 1997 Resveratrol, a polyphenolic compound found in grapes and wine, is an agonist for the estrogen receptor. PNAS 94 14138-14143.

Gotteland M, Desauty G, Delarue JC, Liu L \& May E 1995 Human estrogen receptor messenger RNA variants in both normal and tumor breast tissues. Molecular and Cellular Endocrinology 112 $1-13$.

Henderson BE, Ross R \& Bernstein L 1988 Estrogens as a cause of human cancer: the Richard and Hinda Rosenthal Foundation award lecture. Cancer Research 48 246-253.

Hopp TA \& Fuqua SA 1998 Estrogen receptor variants. Fournal of Mammary Gland Biology and Neoplasia 3 73-83.

Howell A, DeFriend D, Robertson J, Blamey R \& Walton P 1995 Response to a specific antioestrogen (ICI 182,780) in tamoxifen-resistant breast cancer. Lancet 345 29-30.

Jiang SY, Wolf DM, Yingling JM, Chang C \& Jordan VC 1992 An estrogen receptor positive MCF-7 clone that is resistant to antiestrogens and estradiol. Molecular and Cellular Endocrinology 90 $77-86$.

Jordan VC 1998 Antiestrogenic action of raloxifene and tamoxifen: today and tomorrow. Fournal of the National Cancer Institute $\mathbf{9 0}$ 967-971.

Jordan VC 2000 Antiestrogens: clinical applications of pharmacology. Fournal of the Society for Gynecological Investigation $\mathbf{7}$ S47-S48.

Koehorst SG, Jacobs HM, Thijssen JH \& Blankenstein MA 1993 Wild type and alternatively spliced estrogen receptor messenger RNA in human meningioma tissue and MCF-7 breast cancer cells. Fournal of Steroid Biochemistry and Molecular Biology 45 227-233.

Leonessa F, Boulay V, Wright A, Thompson EW, Brunner N \& Clarke R 1992 The biology of breast tumor progression. Acquisition of hormone independence and resistance to cytotoxic drugs. Acta Oncologica 31 115-123.

McGuire WL, Chamness GC \& Fuqua SA 1992 Abnormal oestrogen receptor in clinical breast cancer. European fournal of Cancer 28 309-310.

Madsen MW, Reiter BE, Larsen SS, Briand P \& Lykkesfeldt AE 1997 Estrogen receptor messenger RNA splice variants are not involved in antiestrogen resistance in sublines of MCF-7 human breast cancer cells. Cancer Research 57 585-589. 
Miksicek RJ, Lei Y \& Wang Y 1993 Exon skipping gives rise to alternatively spliced forms of the estrogen receptor in breast tumor cells. Breast Cancer Research and Treatment 26 163-174.

Milla-Santos A, Milla L, Portella J, Rallo L, Pons M, Rodes E, Casanovas J \& Puig-Gali M 2003 Anastrozole versus tamoxifen as first-line therapy in postmenopausal patients with hormone-dependent advanced breast cancer: a prospective, randomized, phase III study. American Fournal of Clinical Oncology 26 317-322.

Murphy LC, Dotzlaw H, Leygue E, Coutts A \& Watson P 1998 The pathophysiological role of estrogen receptor variants in human breast cancer. Fournal of Steroid Biochemistry and Molecular Biology 65 175-180.

Osborne CK, Coronado-Heinsohn EB, Hilsenbeck SG, McCue BL, Wakeling AE, McClelland RA, Manning DL \& Nicholson RI 1995 Comparison of the effects of a pure steroidal antiestrogen with those of tamoxifen in a model of human breast cancer. Fournal of the National Cancer Institute 87 746-750.

Pfeffer U, Fecarotta E \& Vidali G 1995 Coexpression of multiple estrogen receptor variant messenger RNAs in normal and neoplastic breast tissues and in MCF-7 cells. Cancer Research $\mathbf{5 5}$ 2158-2165.

Sluyser M 1994 Hormone resistance in cancer: the role of abnormal steroid receptors. Critical Reviewes in Oncogenesis 5 539-554.
Soule HD \& McGrath CM 1980 Estrogen responsive proliferation of clonal human breast carcinoma cells in athymic mice. Cancer Letters $10177-189$

Thompson EW, Brunner N, Torri J, Johnson MD, Boulay V, Wright A, Lippman ME, Steeg PS \& Clarke R 1993 The invasive and metastatic properties of hormone-independent but hormone-responsive variants of MCF-7 human breast cancer cells. Clinical and Experimental Metastasis Exp Metastasis 11 15-26.

Wang Y \& Miksicek RJ 1991 Identification of a dominant negative form of the human estrogen receptor. Molecular Endocrinology $\mathbf{5}$ 1707-1715.

Wolf DM \& Jordan VC 1994 The estrogen receptor from a tamoxifen-stimulated MCF-7 tumor variant contains a point mutation in the ligand binding domain. Breast Cancer Research and Treatment 31 129-138.

\author{
Received in final form 24 February 2004 \\ Accepted 10 March 2004 \\ Made available online as an \\ Accepted Preprint 23 March 2004
}

